\title{
Zeeman-Doppler imaging of II Peg
}

\author{
Thorsten A. Carroll ${ }^{1}$, Markus Kopf ${ }^{1}$, Klaus G. Strassmeier ${ }^{1}$, \\ Ilya Ilyin ${ }^{1}$ and Ilkka Tuominen ${ }^{2}$ \\ ${ }^{1}$ Astrophysikalisches Institut Potsdam, An der Sternwarte 16, D-14882 Potsdam, Germany \\ email: tcarroll@aip.de \\ ${ }^{2}$ Observatory, University of Helsinki, PO Box 14, FI-00014 Helsinki, Finland
}

\begin{abstract}
We present Zeeman-Doppler images of the active K2 star II Peg for the years 2004 and 2007. The surface magnetic field was reconstructed with our new ZDI code $i M a p$ which provides a full polarized radiative transfer driven inversion to simultaneously reconstruct the surface temperature and magnetic vector field distribution. II Peg shows a remarkable large scale magnetic field structure for both years. The magnetic field is predominantly located at high latitudes and is arranged in active longitudes. A dramatic evolution in the magnetic field structure is visible for the two years, where a dominant and largely unipolar field in 2004 has changed into two distinct and large scale bipolar structures in 2007.
\end{abstract}

Keywords. Stars: activity - stars: magnetic fields - stars: spots - radiative transfer - methods: data analysis

\section{Introduction}

Zeeman-Doppler imaging (ZDI) also known as Magnetic-Doppler imaging (MDI) is a powerful inversion method to reconstruct stellar magnetic surface fields and has significantly contributed to our current understanding of stellar magnetic fields (e.g. Donati et al. 1997; Donati 1999; Kochukhov et al. 2004; Donati et al. 2006, 2007). As ZDI is a non-linear inverse problem it critically depends on the underlying forward modeling and particular attention must be given to a correct modeling of radiative transfer effects (see Carroll et al. this proceeding). In an effort to pursue a more rigorous modeling we have developed our ZDI code $i M a p$ which incorporates a full polarized radiative transfer based inversion (Carroll et al. 2007, 2008). The inverse module is based on either a conjugate gradient or Levenberg-Marquardt method. The regularization that we apply is based on a new local maximum entropy (Carroll et al. 2007). One of the great obstacles for a radiative transfer based interpretation of observed Stokes profiles is the noise level. Although there exist a powerful multi-line reconstruction technique, i.e. the least-square deconvolution method of Donati et al. (1997), the interpretation of the reconstructed mean line profiles is not straight forward. Another method which provides a way of reconstructing individual Stokes line profiles and thus allows a proper radiative transfer modeling was introduced by Carroll et al. (2007); Martínez González et al. (2008) and is based on a principal component analysis reconstruction technique. (see also Carroll et al. this proceeding). Our code was applied to Stokes $I$ and $V$ observations of the K2 subgiant II Pegsi. obtained with the SOFIN spectrograph at the Nordic Optical Telescope (La Palma). The data were recorded in Summer 2004 and 2007 and cover 8 and 10 rotational phases respectively. The original $\mathrm{S} / \mathrm{N}$ of the data were 270 and after the application of our multi-line PCA reconstruction (with 880 spectral lines) we could enhance the S/N of our target lines to 2000 . 

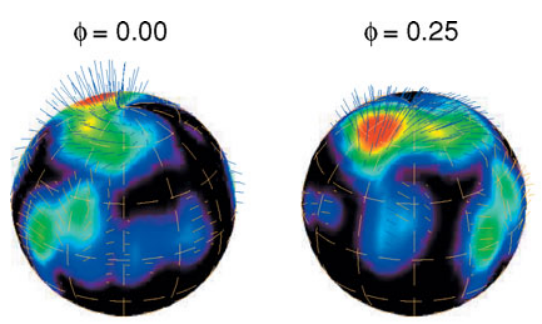

$\phi=0.00$

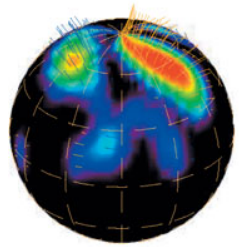

$\phi=0.25$

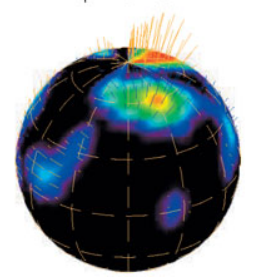

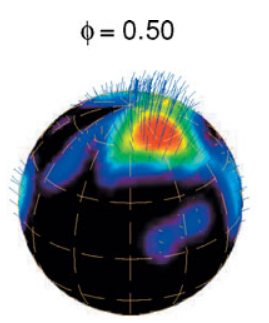

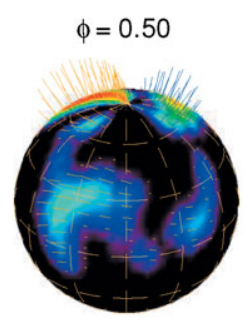

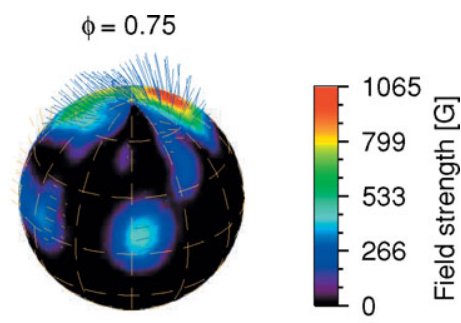

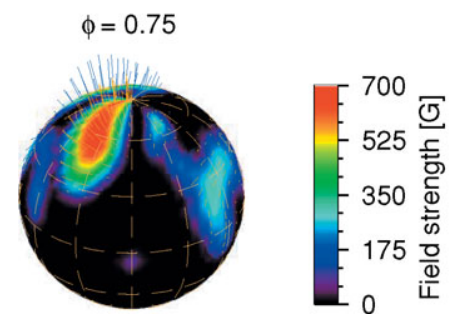

Figure 1. The reconstructed surface magnetic field of II Peg, 2004 (top) and 2007 (bottom). Bright and dark field lines indicate the polarities.

\section{The surface magnetic field of II Peg in 2004 and 2007}

From the reconstruction (figure 1) we can readily identify that the magnetic field of II Peg consist of a small number of large scale magnetic structures which predominantly located at high latitudes and arranged in active longitudes. It is conspicuous that the field is mainly radially oriented an effect which we attribute to our improved $\mathrm{S} / \mathrm{N}$ ratio that allows our inversion to better constrain the field orientation (see also Kochukhov et al. this proceeding). A very interesting evolution can be seen from figure 1, the field structure has changed from a more or less coherent unipolar region in 2004 to two separate field structures of different polarity. This characteristic change of the surface magnetic field topology resembles that of a flip-flop event as found from temperature inversions (Berdyugina 2007) and also found in theoretical modeling of an $\alpha-\Omega$ dynamo (Elstner $\&$ Korhonen 2005). Although, it is to early to confirm the presence of a real flip-flop event from our two year data, this work provides the first real indication of a magnetic reorganization on II Peg.

\section{References}

Berdyugina, S. V. 2007, Mem. Soc. Astr. It. 78, 242

Carroll, T. A., Kopf, M., \& Strassmeier, K. G. 2008, A\& $A$ 488, 781

Carroll, T. A., Kopf, M., Ilyin, I., \& Strassmeier, K. G. 2007, AN 328, 1043

Donati, J.-F., Jardine, M. M., Gregory, S. G., Petit, P., Bouvier, J., Dougados, C., Ménard, F., Cameron, A. C., Harries, T. J., Jeffers, S. V., \& Paletou, F. 2007, MNRAS 380, 1297

Donati, J.-F., Forveille, T., Cameron, A. C., Barnes, J. R., Delfosse, X., Jardine, M. M., \& Valenti, J. A. 2006, Science 311, 633

Donati, J.-F. 1999, MNRAS 302, 457

Donati, J.-F., Semel, M., Carter, B. D., Rees, D. E., \& Collier Cameron, A. 1997, MNRAS 291, 658

Elstner, D. \& Korhonen, H. 2005, AN 326, 278

Kochukhov, O., Bagnulo, S., Wade, G. A., Sangalli, L., Piskunov, N., Landstreet, J. D., Petit, P., \& Sigut, T. A. A. 2004, A\&\&A 414, 613

Martínez González, M. J., Asensio Ramos, A., Carroll, T. A., Kopf, M., Ramírez Vélez, J. C., \& Semel, M. 2008, A\& $A$ 486, 637 